\title{
Efficient Couplers for Photonic Crystal Waveguides
}

\author{
K. Dossou ${ }^{1}$, L. C. Botten ${ }^{1}$, C. M. de Sterke ${ }^{2}$, R. C. \\ McPhedran $^{2}$, A. A. Asatryan ${ }^{1}$, S. Chen ${ }^{2}$ and J. Brnovic ${ }^{2 *}$
}

\begin{abstract}
We use two-dimensional simulations to study the design of tapers to provide efficient, low reflection coupling between a waveguide in a two-dimensional photonic crystal (PC) and free space. We find that, largely independent of the PC parameters, or of the length and width of the tapered region, the same type of concave, horn-shaped tapering profile is optimal for coupling from the waveguide into free space, and significantly out-performs the widely-used linear taper. We also find that optimal tapers can radiate nearly Gaussian beams, and therefore they can also provide efficient coupling of Gaussian beams from free space into the PC waveguide. These properties are better exhibited by rod-type PCs with $E_{z}$ polarization than by hole-type PCs with $H_{z}$ polarization. This study of taper couplers exemplifies a design strategy for photonic circuits which optimizes positioning of the cylinders immediately surrounding the light path, and then builds the rest of the crystal structure around these cylinders .
\end{abstract}

\footnotetext{
${ }^{* 1}$ Centre for Ultrahigh-Bandwidth Devices for Optical Systems (CUDOS) and Department of Mathematical Sciences, University of Technology Sydney, PO Box 123, NSW 2007 Australia.

${ }^{2}$ CUDOS and School of Physics, University of Sydney 2006 Australia.
} 


\section{INTRODUCTION}

Photonic crystal waveguides (PCWs), formed by the inclusion of line defects in an otherwise perfect photonic crystal (PC), have emerged as a promising application of these structures. One of the key issues in their practical use is the coupling to other guided wave structures, or to free space. It is well known, for example, that a suddenly terminated PCW exhibits significant reflection, and that the reflections off two such terminations lead to Fabry-Perot like fringes [1]. This can be avoided by tapering the transition, a topic that has attracted much interest [2]-[13]. In this previous work, a variety two-dimensional problems are considered, such as the coupling between a dielectric waveguide and PCWs $[2,7,10]$, the coupling of two slab waveguides via a PCW [3], the coupling from a PCW to free space $[4,5,8,9]$, and the coupling between two different PCWs [6]. A recent study [14] reports the comparison between two-dimensional theory, three-dimensional theory and experiment for a device comprising a taper, a Y-splitter and two bends, with the measured device transmittance being $70 \%$.

It is apparent in the literature is that fabrication technology and computational methods have improved rapidly, permitting the study of sophisticated and efficient coupler designs. For example, Bienstman et al. [11] demonstrate an input coupler between a dielectric waveguide and a PCW with a transmission over $90 \%$ over a wide frequency range $(\Delta f / f>11 \%)$. Pottier et al. [10] study computationally and experimentally a taper coupler between a Gaussian beam in free space and a PCW, and show that a transmission of $98.4 \%$ can be obtained for a fixed frequency, with the efficiency exceeding $95 \%$ for a frequency range of over $1.8 \%$.

The common element in this work is that taper designs are chosen from a somewhat limited range of profiles: increasingly, linear tapers are being used [10, 12], but a recent and quite successful design [13] achieves good transmission by optimizing the choice of where the input face of the photonic crystal is placed. Kramper et al [15] study the radiation by PCW's into free space, and show the importance of the termination of the PC on the output waveform.

As far as we know, no investigations have regarded the taper as being defined by a function which may be varied continuously, so as to optimize transmission for a taper of given length. Here we select tapers from a general class of smooth shapes to improve the coupling between a two-dimensional PCW and free space, similar to the work of Happ et 
al. [4], and in keeping with the adiabatic theorem of Johnson et al. [16]. We consider a variety of tapers in both rod-type and air-hole type PC. We find numerically that, almost independently of the details of the geometry, the same type of concave, horn-shaped taper always leads to an optimal coupling. By optimal, of course, we mean the best solution among the set of all tapers from the given family of profiles. Thus this implies a general solution for the coupling between a PCW and free space.

We also investigate the amplitude and phase distribution of the beam radiated into free space by the optimal taper. We show that the beam can in fact be very close to being pure Gaussian, in contrast to non-optimal tapers. From the Reciprocity Theorem [17], we thus conclude that the shape which is optimal for coupling a waveguide mode into free space is also optimal for the reverse process. We provide examples substantiating this deduction.

It should be stressed that our main aim is not to present an optimized solution to coupling between photonic crystal waveguides in two-dimensions and free space as an end in its own right. Rather, we choose this coupling problem as a mechanism to illustrate that present optimized solutions could use a wider range of geometrical possibilities than is current practice. Specifically, we show that good performance in compact devices can be achieved by designing from the light path outwards, rather than from the photonic crystal inwards: we start building the photonic crystal circuit by optimally placing the cylinders which define the light path, and their nearest neighbours. We then add further cylinders in such a way as to revert gradually towards the underlying photonic crystal structure. For the implementation of this strategy in the case of the taper coupler, see Section IIB.

In Section 2, we discuss the numerical methods we use to calculate the energy transmitted from a PCW into free space through a taper, as well as the construction of the family of smooth taper profiles we used in our investigation of optimal taper shapes (with details given in the Appendix). In Section 3, we study the coupling between a PCW and free space, commenting on the efficiency of taper coupling as a function both of taper shape and taper length. In Section 4 we discuss how an optimal fit can be achieved between the wave coupled by a given taper into free space and a Gaussian wave, and use this to analyze beams radiated by PCWs. In Section 5, we give numerical examples confirming that the taper shapes we have identified to be optimal for coupling a PCW mode into free space are also optimal for the reverse process. Finally, in Section 6, we draw conclusions from this work. 


\section{THEORETICAL AND NUMERICAL METHOD}

In our theoretical approach we think of the PC as consisting of "layers," each of which can be considered to be a diffraction grating, described by scattering matrices. In our previous work on nonuniform PCs $[18,19]$, subsequent constituent gratings are identical to each other, so that the structure consists of sections that are perfectly periodic, so that Bloch's theorem can be used to describe the field. Here this approach is impractical since in the taper region all constituent gratings are different and the periodicity is lost. In this section we therefore briefly outline our calculational method.

\section{A. Theory of Taper Couplers in Photonic Crystals}

\section{General Framework}

We consider the two-dimensional structures shown in Fig. 1 comprising semi-infinite photonic crystal waveguides in both square and hexagonal lattices and a taper region, shaped in some appropriate way to apodize the discontinuity between the waveguide and free space. In our model, each of the constituent layers, of both the semi-infinite guide and the taper, is treated as a diffraction grating having a supercell that contains the functional elements (i.e., guide, taper), and chosen to be sufficiently large to ensure that corresponding elements in adjacent supercells are effectively isolated from one another by strong attenuation associated with operating in a band gap of the bulk crystal. With a supercell period of length $D$, the grating generates diffraction orders or channels whose propagation directions $\theta_{s}$ are given by the diffraction grating equation

$$
k \sin \theta_{s}=\alpha_{s}=\alpha_{0}+\frac{2 \pi s}{D},
$$

where $k$ is the wavenumber in the background. For analytic and numerical convenience [19], we impose a periodic boundary condition $\left(\alpha_{0}=0\right)$ on the supercell. In Section 5 we operate the device with a Gaussian beam. The accurate representation of this beam in a plane wave series requires a suitably large number of terms. Accordingly, the supercell must be chosen sufficiently large to generate a finely spaced plane wave basis that closely approximates the beam, while simultaneously meeting the adjacent supercell isolation requirement.

The diffraction properties of each of the gratings are represented by plane wave reflection 
$(\widetilde{\boldsymbol{R}})$ and transmission $(\widetilde{\boldsymbol{T}})$ scattering matrices, the elements of which are, for example, $\widetilde{R}_{p q}-$ the complex amplitude of the plane wave reflected into the channel $p$ associated with an angle $\theta_{p}$ due to an incident plane wave of unit amplitude in channel $q$ (with angle $\theta_{q}$ ). While scattering matrices can be computed using a variety of methods, here we use multipole techniques [20, 21] and the finite element method [22].

For a taper composed of a sequence of $L$ layers, we compute scattering matrices $\left(\widetilde{\boldsymbol{R}}_{l}, \widetilde{\boldsymbol{T}}_{l}\right)$ for layers $l=1,2, \ldots, L$, enumerating these from bottom to top. We can then form the reflection and transmission matrices for the taper associated with incidence from below

$$
\widetilde{\boldsymbol{R}}_{\text {taper }}^{\prime}=\widetilde{\boldsymbol{R}}_{1, L}, \quad \widetilde{\boldsymbol{T}}_{\text {taper }}^{\prime}=\widetilde{\boldsymbol{T}}_{1, L}
$$

using backward recursion

$$
\begin{aligned}
\widetilde{\boldsymbol{R}}_{l-1, L} & =\widetilde{\boldsymbol{R}}_{l-1}+\widetilde{\boldsymbol{T}}_{l-1} \widetilde{\boldsymbol{R}}_{l, L}\left(\boldsymbol{I}-\widetilde{\boldsymbol{R}}_{l-1} \widetilde{\boldsymbol{R}}_{l, L}\right)^{-1} \widetilde{\boldsymbol{T}}_{l-1} \\
\widetilde{\boldsymbol{T}}_{l-1, L} & =\widetilde{\boldsymbol{T}}_{l, L}\left(\boldsymbol{I}-\widetilde{\boldsymbol{R}}_{l-1} \widetilde{\boldsymbol{R}}_{l, L}\right)^{-1} \widetilde{\boldsymbol{T}}_{l-1},
\end{aligned}
$$

commencing with $\widetilde{\boldsymbol{R}}_{L, L}=\widetilde{\boldsymbol{R}}_{L}$ and $\widetilde{\boldsymbol{T}}_{L, L}=\widetilde{\boldsymbol{T}}_{L}$. For incidence from above, the corresponding taper scattering matrices are $\widetilde{\boldsymbol{R}}_{\text {taper }}=\widetilde{\boldsymbol{R}}_{L, 1}$ and $\widetilde{\boldsymbol{T}}_{\text {taper }}=\widetilde{\boldsymbol{T}}_{L, 1}$, which are computed in a similar manner, but now with forward recursion.

In the waveguide region, we represent the field in its natural Bloch mode basis $[18,19]$. To do so, we consider the plane wave representation of the field on the interfaces at $y=y_{j}$ between adjacent layers $j=1,2$

$$
V_{f}^{(j)}(\mathbf{r})=\sum_{s=-\infty}^{\infty} \chi_{s}^{-1 / 2}\left[f_{(-) s}^{(j)} e^{-i \chi_{s}\left(y-y_{j}\right)}+f_{(+) s}^{(j)} e^{i \chi_{s}\left(y-y_{j}\right)}\right] e^{i \alpha_{s} x},
$$

with $\chi_{s}=\sqrt{k^{2}-\alpha_{s}^{2}}=k \cos \theta_{s}$ (c.f. Eq.(1)). The expansion comprises upward and downward propagating plane waves, with amplitudes contained in the vectors $\boldsymbol{f}_{+}^{(j)}$ and $\boldsymbol{f}_{-}^{(j)}$, respectively.

The Bloch modes of an infinite waveguide are now derived by solving an eigenvalue problem for the transfer matrix $\mathcal{T}$ that relates fields on either side of a grating layer according to $\boldsymbol{f}_{2}=\boldsymbol{T} \boldsymbol{f}_{1}$. The vertical periodicity imposes the Bloch condition $\boldsymbol{f}_{2}=\mu \boldsymbol{f}_{1}$, with Bloch factor $\mu$, so that

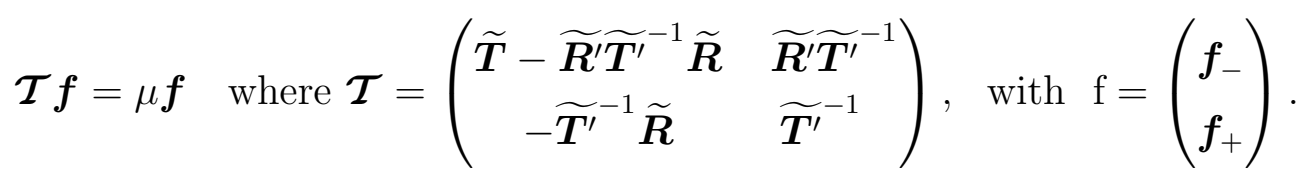


Here $\widetilde{\boldsymbol{R}}$ and $\widetilde{\boldsymbol{T}}$ are reflection and transmission matrices for a single layer of the bulk crystal for incidence from above, while $\widetilde{\boldsymbol{R}^{\prime}}$ and $\widetilde{\boldsymbol{T}^{\prime}}$ are the corresponding matrices for incidence from below.

The solutions of eigenvalue equation (6) may be partitioned into modes which are downward and upward propagating. For propagating modes, that have eigenvalues $|\mu|=1$, the partitioning is done according to direction of energy flow, while for evanescent modes $(|\mu| \neq 1)$, the partitioning occurs according to the direction of decay of the field. The transfer matrix may be diagonalized [19]

$$
\mathcal{T}=\mathcal{F} \mathcal{L} \mathcal{F}^{-1}, \quad \text { where } \mathcal{F}=\left(\begin{array}{cc}
\boldsymbol{F}_{-} & \boldsymbol{F}_{-}^{\prime} \\
\boldsymbol{F}_{+} & \boldsymbol{F}_{+}^{\prime}
\end{array}\right) \quad \text { and } \quad \mathcal{L}=\left(\begin{array}{cc}
\boldsymbol{\Lambda} & \mathbf{0} \\
\mathbf{0} & \boldsymbol{\Lambda}^{\prime}
\end{array}\right)
$$

The columns of matrix $\mathcal{F}$ comprise the eigenvectors constituting the Bloch modes. Its left partition contains the downward propagating modes, whereas the right partition (with quantities distinguished by a superscripted prime (') contains the upward propagating modes. The diagonal matrix $\mathcal{L}$ comprises the eigenvalues $\mu$ [19]. For PCs with a square or rectangular lattice, $\boldsymbol{F}_{ \pm}^{\prime}=\boldsymbol{F}_{\mp}[19]$. We normalize the Bloch mode matrix $\mathcal{F}$ so that modes carry unit energy, thus reducing energy flux calculations to the computation of the square magnitude of the relevant coefficient(s) in the Bloch mode expansion [19].

We turn now to the calculation of the reflection and transmission coefficients for the two ways in which the guide is to be operated: the propagation of a wave from the guide into free space, and vice versa.

\section{Waveguide to free space formulation}

We consider fields at the interface between the waveguide and the taper. Below the interface, the fields comprise upward propagating modes (designated by a vector of Bloch mode coefficients $\left.\boldsymbol{c}_{+}\right)$incident on the interface and downward propagating modes $\left(\boldsymbol{c}_{-}\right)$reflected from the interface. Thus, at the interface, the field is expressed in terms the plane wave expansion (5) with upward and downward components $\left(\boldsymbol{g}_{ \pm}\right)$, where

$$
\left(\begin{array}{l}
\boldsymbol{g}_{-} \\
\boldsymbol{g}_{+}
\end{array}\right)=\left(\begin{array}{l}
\boldsymbol{F}_{-} \\
\boldsymbol{F}_{+}
\end{array}\right) \boldsymbol{c}_{-}+\left(\begin{array}{c}
\boldsymbol{F}_{-}^{\prime} \\
\boldsymbol{F}_{+}^{\prime}
\end{array}\right) \boldsymbol{c}_{+}=\mathcal{F} \boldsymbol{c}
$$


The interaction of the plane wave fields $\boldsymbol{g}_{ \pm}$and the field $\boldsymbol{t}$ transmitted into free space is characterized by the taper reflection and transmission matrices:

$$
\boldsymbol{g}_{-}=\boldsymbol{R}_{\text {taper }}^{\prime} \boldsymbol{g}_{+}, \quad \boldsymbol{t}=\boldsymbol{T}_{\text {taper }}^{\prime} \boldsymbol{g}_{+}
$$

Solving Eqs (8) and (9), we find expressions for the reflection $\left(\boldsymbol{R}_{\mathrm{gf}}\right)$ and transmission $\left(\boldsymbol{T}_{\mathrm{gf}}\right)$ matrices of the "guide-taper-free space" system, defined by

$$
c_{-}=R_{\mathrm{gf}} c_{+}, \quad t=T_{\mathrm{gf}} c_{+}
$$

We thus derive

$$
\begin{aligned}
\boldsymbol{R}_{\mathrm{gf}} & =\left(\boldsymbol{F}_{-}\right)^{-1}\left(\boldsymbol{I}-\boldsymbol{R}_{\mathrm{taper}}^{\prime} \boldsymbol{R}_{g}\right)^{-1}\left(\boldsymbol{R}_{\mathrm{taper}}^{\prime}-\boldsymbol{R}_{g}^{\prime}\right) \boldsymbol{F}_{+}^{\prime}, \\
\boldsymbol{T}_{\mathrm{gf}} & =\boldsymbol{T}_{\text {taper }}^{\prime}\left(\boldsymbol{I}-\boldsymbol{R}_{g} \boldsymbol{R}_{\text {taper }}^{\prime}\right)^{-1}\left(\boldsymbol{I}-\boldsymbol{R}_{g} \boldsymbol{R}_{g}^{\prime}\right) \boldsymbol{F}_{+}^{\prime} .
\end{aligned}
$$

Here $\boldsymbol{R}_{g}=\boldsymbol{F}_{+}\left(\boldsymbol{F}_{-}\right)^{-1}$ and $\boldsymbol{R}_{g}^{\prime}=\boldsymbol{F}_{-}^{\prime}\left(\boldsymbol{F}_{+}^{\prime}\right)^{-1}$ are the plane wave reflection matrices of semiinfinite PCs, respectively corresponding to incidence from above and below [19]. For an incident modal field $\boldsymbol{c}_{+}$, the reflected modal field $\boldsymbol{c}_{-}$and the transmitted plane wave field $\boldsymbol{t}$ then follow from Eqs (10). Since the plane wave and waveguide bases are normalized their reflection and transmission coefficients satisfy normalization conditions as well.

\section{Free space to waveguide formulation}

We now consider an incident plane wave field $\boldsymbol{\delta}$ incident onto the structure from above, where the elements are the amplitudes of each plane wave in the incident field. This leads to an upward going reflected plane wave field $\boldsymbol{r}$ above the taper, a plane wave field with upward and downward components $\boldsymbol{g}_{ \pm}$at the interface, and a downward, transmitted Bloch mode field $\boldsymbol{c}_{-}$into the waveguide. The relationships between these quantities are expressed through the various scattering matrices as

$$
\begin{aligned}
\boldsymbol{r} & =\boldsymbol{R}_{\mathrm{taper}} \boldsymbol{\delta}+\boldsymbol{T}_{\mathrm{taper}}^{\prime} \boldsymbol{g}_{+}, \quad \boldsymbol{g}_{-}=\boldsymbol{T}_{\mathrm{taper}} \boldsymbol{\delta}+\boldsymbol{R}_{\mathrm{taper}}^{\prime} \boldsymbol{g}_{+} \\
\boldsymbol{g}_{+} & =\boldsymbol{R}_{g} \boldsymbol{g}_{-}, \quad \boldsymbol{c}_{-}=\boldsymbol{T}_{g} \boldsymbol{g}_{-}=\left(\boldsymbol{F}_{-}\right)^{-1} \boldsymbol{f}_{-}
\end{aligned}
$$

Introducing scattering matrices $\boldsymbol{R}_{\mathrm{fg}}$ and $\boldsymbol{T}_{\mathrm{fg}}$ defined by

$$
\boldsymbol{r}=\boldsymbol{R}_{\mathrm{fg}} \boldsymbol{\delta}, \quad \boldsymbol{c}_{-}=\boldsymbol{T}_{\mathrm{fg}} \boldsymbol{\delta}
$$


and solving Eqs (13)-(14), we deduce

$$
\begin{aligned}
\boldsymbol{R}_{\mathrm{fg}} & =\boldsymbol{R}_{\text {taper }}+\boldsymbol{T}_{\text {taper }}^{\prime} \boldsymbol{R}_{g}\left(\boldsymbol{I}-\boldsymbol{R}_{\text {taper }}^{\prime} \boldsymbol{R}_{g}\right)^{-1} \boldsymbol{T}_{\text {taper }}, \\
\boldsymbol{T}_{\mathrm{fg}} & =\left(\boldsymbol{F}_{-}\right)^{-1}\left(\boldsymbol{I}-\boldsymbol{R}_{\text {taper }}^{\prime} \boldsymbol{R}_{g}\right)^{-1} \boldsymbol{T}_{\text {taper }}
\end{aligned}
$$

Thus, for an incident plane wave field $\boldsymbol{\delta}$, we compute the reflected plane wave field $\boldsymbol{r}$ and the transmitted waveguide modal field $\boldsymbol{c}_{-}$from (15), which, as before, satisfy an energy conservation relation.

\section{B. Numerical Implementation}

All tapers considered become monotonically wider between the semi-infinite PCW and free space, with the waveguide always being formed by omission of a single line of cylinders (i.e, being a W1 guide). We studied both step-wise tapers, in which all inclusions appear in their positions in the regular structure and the taper consists of a region in which some of the inclusions are removed, leading to a taper with jagged edges [4], and smooth tapers, in which the inclusions can appear at any transverse position (see Fig. 1) [9-11]. Since the inclusions are now removed from their regular positions in the PCW, this requires a strategy to place the other inclusions in each grating. That adopted here, illustrated in Figs. 1, uses the fact that the light is confined most strongly in the part of the PC immediately around the PCW [23], and therefore that it is important to preserve smooth lines on and adjacent to the taper walls (designing from the light path outwards). Hence the distance between the two inclusions closest to the taper opening is kept at $d$, with subsequent inclusions separated by $d^{\prime}$, such that $d^{\prime}$ is as close as possible to $d$, while consistent with the supercell period $D$; $d^{\prime}$ generally differs in each of the gratings. Though we did consider tapers with jagged edges, we found that the transmission through these tapers is inferior to that of smooth tapers, consistent with the results of Kwan et al. [23]. We therefore do not consider these tapers here further.

We now introduce the parameters that describe the taper. Let $d_{x}$ and $d_{y}$ denote the lateral period and vertical thickness of the perfect PC layer. The integer $L$ represents the number of taper layers; $W_{\text {base }} d_{x}$ and $W d_{x}$ are respectively the width at the lower and upper ends of the taper. In the case of a square lattice we have $d_{x}=d, d_{y}=d$ and $W_{\text {base }}=2$, while for the hexagonal lattice $d_{x}=d \sqrt{3}, d_{y}=d / 2$ and $W_{\text {base }}=1$, as illustrated in Fig. 1. 
(a)

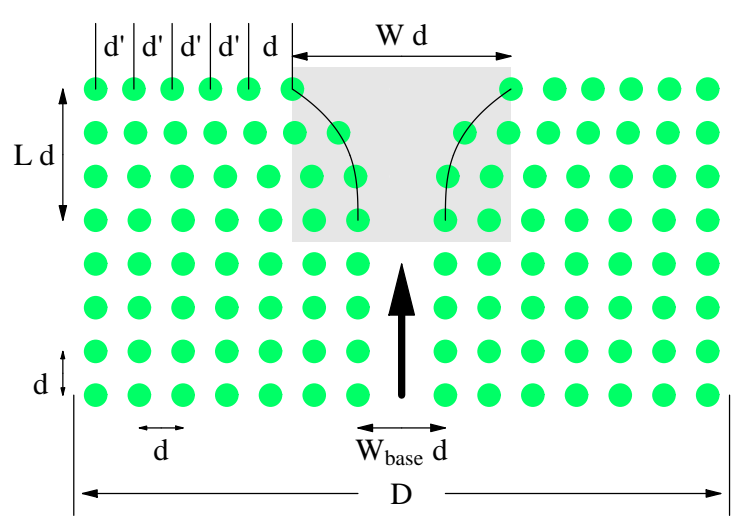

(b)

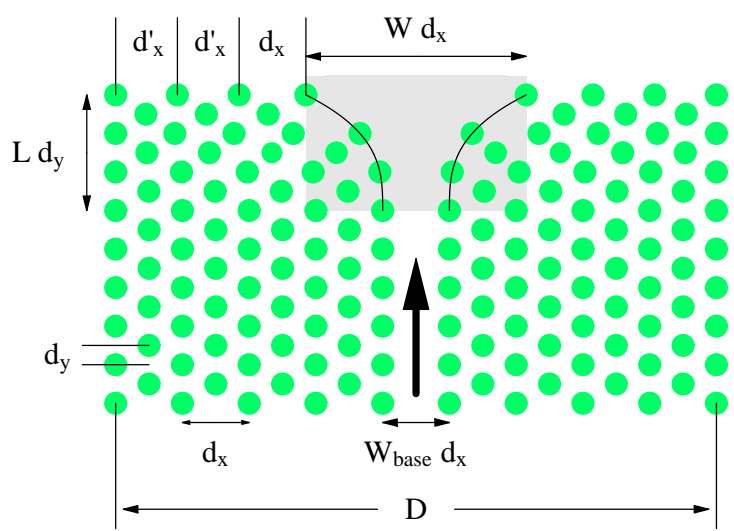

FIG. 1: Schematics of the (a) square and (b) hexagonal lattice tapers. Light is incident from a PCW (large arrow) into free space via a taper of $L$ layers and width $W d$ (shaded rectangle). The PC is considered to consist of grating with supercell period $D$. The solid curves correspond to Eq. (18) specifying the positions of the scatterers.

The width of the taper is $w(y) d_{x}$, defined from the centre of the inclusions, where

$$
w(y)=W_{\text {base }}+\left(W-W_{\text {base }}\right)\left(\frac{y}{L d_{y}}\right)^{\xi}, \quad \text { for } y \in\left[0, L d_{y}\right]
$$

where $y$ runs along the taper length, starting at the lower end of the shaded region in Fig. 1 where the width is $W_{\text {base }} d_{x}$. Thus, for a square lattice, $W=2$ corresponds to an untapered waveguide, so that the distance between the centres of the inclusions at the taper mouth is $2 d_{x}=2 d$. For a guide in a square lattice, the width of the exit of the taper is $W d$, as shown in Fig. 1. The positive parameter $\xi$ determines the taper shape: for $\xi=1$ the taper is linear, for $\xi>1$ it is concave (horn-shaped; as in Fig. 1), while for $\xi<1$ it is convex. Note that previous designs for optimized couplers have all used $\xi=1$.

As discussed in the Appendix, the situation is more complex for hexagonal lattices since even the untapered guide is not uniform. At the mouth of the waveguide (at $y=0$ ), the centre-to-centre separation of the cylinders is $W_{\text {base }} d_{x}=d \sqrt{3}$. We proceed to fit the centre of the scatterer in each even layer by Eq. (18) and then set the width of its successor (odd) layer by adding $d_{x}=d \sqrt{3}$. The width of the exit of the taper is thus either $W d_{x}$, if $L$ is even, or $(W+1) d_{x}$ for odd $L$ odd. This distinction is made explicit, as necessary, in the figure captions, with further details given in the Appendix. 


\section{Method Parameters and Accuracy}

The supercell period used in our calculations is $D=21 d$, and the diffraction orders used for the plane wave expansion range from -22 to 22 . Where the scattering matrices are computed using the multipole method [20, 21], multipole expansions have Bessel function orders in the range $\left[-N_{B}, N_{B}\right]$. The truncation parameter $N_{B}$ is chosen typically to be 7 in order to obtain good convergence of Bessel function expansions in the wavelength range of interest. The multipole parameters were chosen after convergence studies of the effects of increasing method parameters on physically important quantities. We also verified them by comparing the results with those from a standard quadratic finite element method [22]. To do so, the surface of each supercell is represented by a finite element mesh with around 11000 triangles and 23000 points and the meshes can vary from one layer to another. The differences between the finite element method results and the multipole results are very small (less than $0.1 \%$ for the wavelengths of interest) and are essentially not visible on the transmittance contour maps. When we use more refined meshes or increase the number of plane wave orders, the changes in the finite element results are negligible. This high accuracy enables us to study the behavior of couplers in regions where the transmission is very high (e.g., above $99.5 \%)$

We consider two types of PCs. The first, PC1, is a rod-type PC for $E_{z}(\mathrm{TM})$ polarization (electric field along the cylinders), consisting of cylinders of radius $a=0.3 d$, and refractive index $n_{c}=3$ arranged in a square array, in a background of refractive index $n_{b}=1$. This PC has a band gap for wavelengths $\lambda$ such that $2.978 d<\lambda<3.774 d$. However, within this gap, the W1 waveguide supports a mode with a cutoff at $\lambda=3.496 d$, so the relevant wavelength range for our study is $2.978 d<\lambda<3.496 d$. We also show results for this PC with slightly changed parameters to demonstrate that our results are valid for a range of PCs. The second PC we study, PC2, is operated with $H_{z}$ (TE) polarization, and has low index cylinders $\left(n_{c}=1\right)$ of radius $a=0.3 d$ in a hexagonal array in a high-index background with $n_{b}=3$ [24]. It has a bandgap for $3.279 d<\lambda<4.199 d$. The cutoff wavelength of the fundamental waveguide mode is $4.090 d$, and the waveguide is multimode for 3.558d $<\lambda<3.766 d$ and single mode elsewhere in the gap. We give results for the first single mode operating range $3.766 d<\lambda<4.090 d$ since near the cutoff the unwanted reflection effect is stronger, and thus the use of a taper is more important in that region. 


\section{OPTIMAL COUPLING FROM PCWS INTO FREE SPACE}

We first consider the transmission properties from the fundamental waveguide mode of the PCW in PC1 into free space, as a function of the length and shape of the tapers. Transmittance for $E_{z}$ polarization and tapers of total width $W=4$ and for taper lengths $L$ ranging from $L=4$ to $L=8$ are shown in Figs 2, for various taper shapes $\xi$, and for the wavelengths of interest. The contour plots have levels ranging from very high values (0.995) down to low values (0.70) reflecting poor taper performance. The waveguide tapers exhibit very high transmittances for horn-shaped tapers $\xi>1$. In this and many other examples we have studied, optimal transmittance tends to occur for tapers with $\xi \approx 2-3$. Note from Fig. 2 that linear tapers $(\xi=1)$ have good performance, but to achieve this the taper has to be longer than for a comparable performance from a taper with $\xi \approx 2-3$. For tapers of optimal shape, transmittance and wavelength coverage both increase with taper length, with a length of six periods having transmittances not significantly below those from longer tapers. The fractional wavelength interval over which the optimal $L=6$ layers taper provides a transmittance over $99.5 \%$ is around $12 \%$, superior to the best values reported $[10,11]$, which correspond to linear tapers with $L=10$ and $L=22$ layers, respectively.

Figure 3 shows the transmittance for tapers of width $W=8$. The main difference from Fig. 2 is that longer tapers are needed to achieve comparable performance with $W=4$ structures: $L=8$ layers in Fig. 3 is roughly comparable with $L=6$ layers in Fig. 2. Once again the optimal tapers have $\xi \approx 2-3$, and even a length of $L=9$ layers is insufficient for a linear taper to provide a transmittance of 0.995. In Fig. 4, we show the effect of decreasing cylinder radius (a) or cylinder refractive index (b) on the performance of optimal tapers $(\xi=3, L=6$ layers, $W=4)$. Both plots show trends and performance that are similar to those in Fig. 2.

Note from Figs. 2-4 that, as expected, the transmittance vanishes as the mode approaches cut-off at $\lambda=3.496 d$ irrespective of the inclusion of a taper. Note however also from the contours that are moving upwards, that tapers with $\xi \approx 2-3$ perform substantially better than straight tapers as cut-off is approached. This is illustrated in Fig. 5, which compares the transmissivity and reflectivity (inset) versus wavelength for an untapered waveguide (dashed curve), a linearly tapered waveguide (long-dashed curve), and an optimally tapered waveguide (solid curve). The improved performance of horn-shaped tapers close to cut-off 

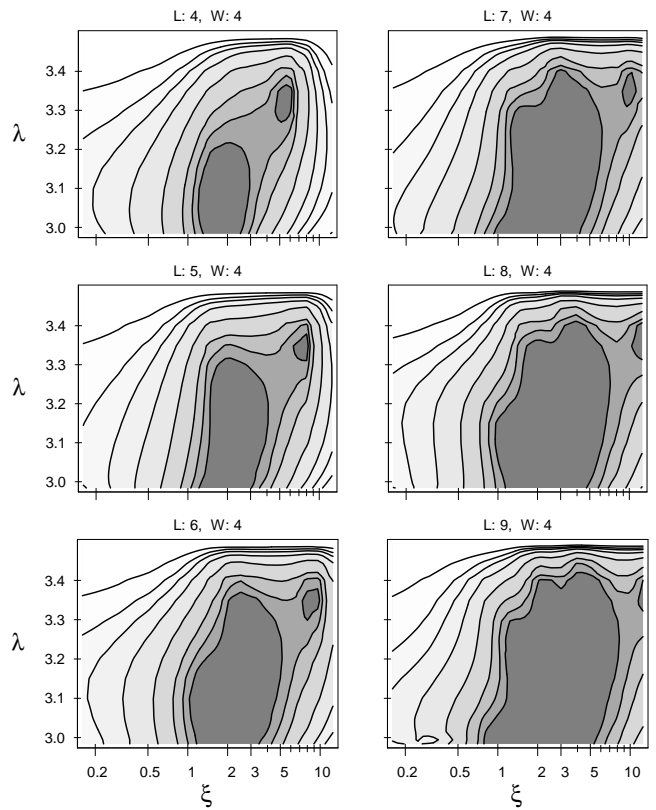

FIG. 2: Transmittance in PC1 through a tapers of width $W=4$ into free space, for taper lengths $L=4-9$ layers. The contour levels correspond to transmittances of $0.995,0.99,0.98,0.95,0.90$, $0.85,0.80$ and 0.70 , decreasing from the region around $\xi \simeq 2$ outwards.
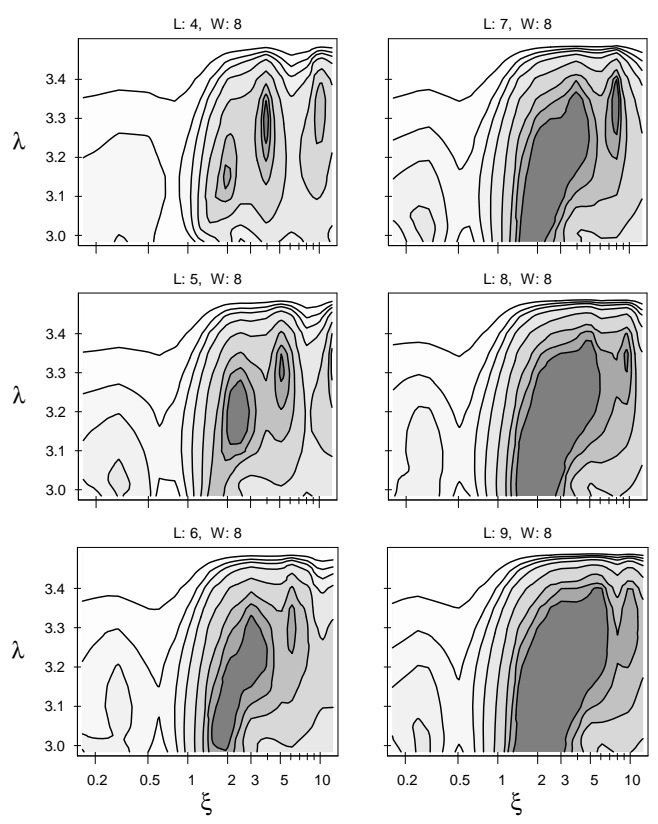

FIG. 3: As Fig. 2 but for $W=8$. 

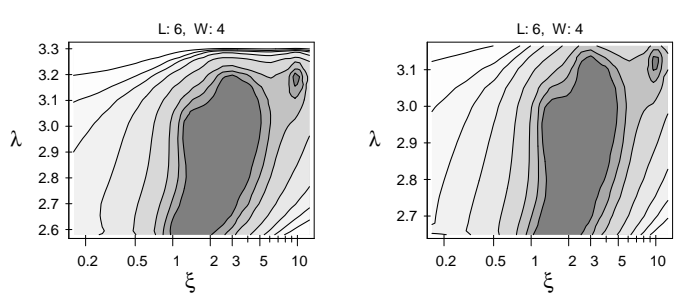

FIG. 4: Transmittance from waveguides in PCs similar to PC1 with taper length $L=6$ layers and width $W=4$ into free space (a) $a=0.25$ and $n_{c}=3.0$, and (b) $a=0.30$ and $n_{c}=2.5$ (right), and with other parameters unchanged.

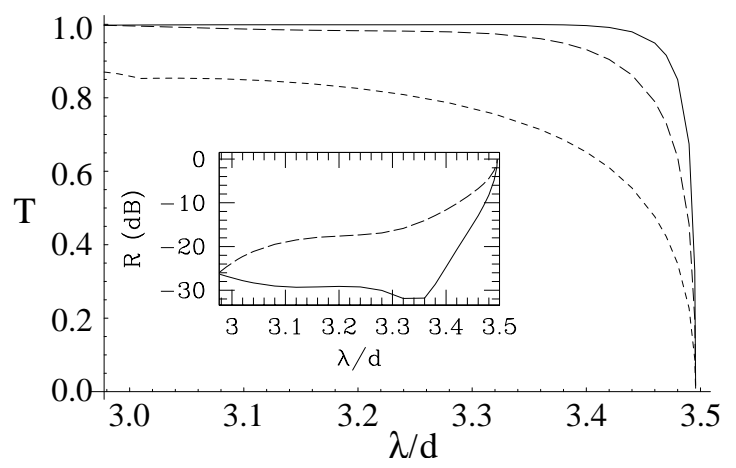

FIG. 5: Transmittance versus wavelength in the single-mode part of the band gap in PC1 for an untapered PCW (short-dashed curve), for a straight taper $(\xi=1)$ with $L=7$ and $W=4$ (long-dashed curve), and for the optimized taper with $L=7 d$ and $W=4 d$ and $\xi=3$ (solid curve). The inset shows the same data expressed as reflection in $\mathrm{dB}$ for the straight (dashed line) and the optimized tapers (solid line).

is particularly significant since this is the regime of slow light propagation, which is a topic of strong interest.

We next show two examples for PC2 for tapers with widths $W=3$ (Figs 6 ) and $W=$ 4 (Figs 7). Both examples show that the combination of high transmittance and good bandwidth can be achieved for this geometry. Once again, the horn-shaped tapers with $\xi \approx 2-3$ have good performance, but there is less regularity in the contours than in Figs $2-4$. In some cases, this may be the result of adjustments in cylinder position made by the taper design algorithm as $\xi$ varies, but it may well reflect the greater intrinsic $H_{z}$ polarization sensitivity to small changes in geometry which is well known in diffraction grating physics [17]. A feature which catches the eye in Fig. 6 is the drop in bandwidth of the very high 

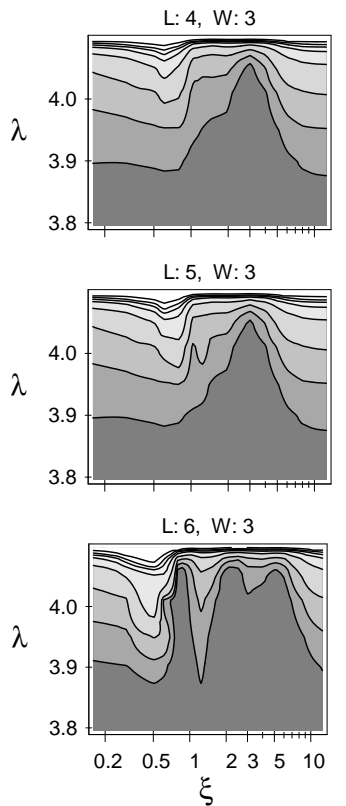

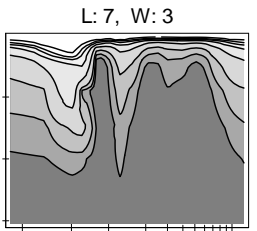

L: $8, \mathrm{~W}: 3$

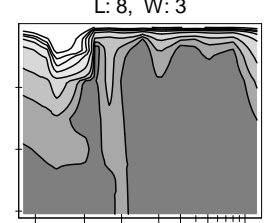

$\mathrm{L}: 9, \mathrm{~W}: 3$

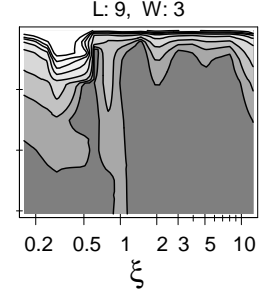

FIG. 6: Transmittance from waveguides in PC2 through tapers of lengths $L=4-9$ layers and $W=3$ into free space. For $L=4,6,8$ the width of the taper mouth is $W d_{x}=3 \sqrt{3} d$ while for $L=5,7,9$ it is $(W+1) d_{x}=4 \sqrt{3} d$.

transmission contours for the linear taper region near $\xi=1$, but it should be noted that this effect disappears for the third contour, associated with the transmittance range around 98\%. An interesting difference between PC1 and PC2 is that for the latter there is not the monotonic improvement in high transmittance bandwidth with taper length $L$ that there is for the former. Indeed, in Figs. 6 and 7, there is no significant improvement in transmittance for $L$ exceeding 6 layers.

\section{BEAM SHAPE FOR RADIATION FROM PCWS}

Having studied the coupling of energy from PCW into free space, we now turn to a second question: the determination of the beam in free space radiated by the waveguide. It is often desirable that this beam be close to Gaussian in profile and here we quantify the degree to which the beam radiated into free space resembles this shape. We commence by describing the method we use to analyze this beam. All waveguides considered here are single mode and thus we prescribe the incident mode vector $\boldsymbol{c}_{-}$with elements $c_{m}^{(-)}=\delta_{m 1}$, indicating that there is unit amplitude (and unit flux) in this mode, labelled as $m=1$. The resulting 

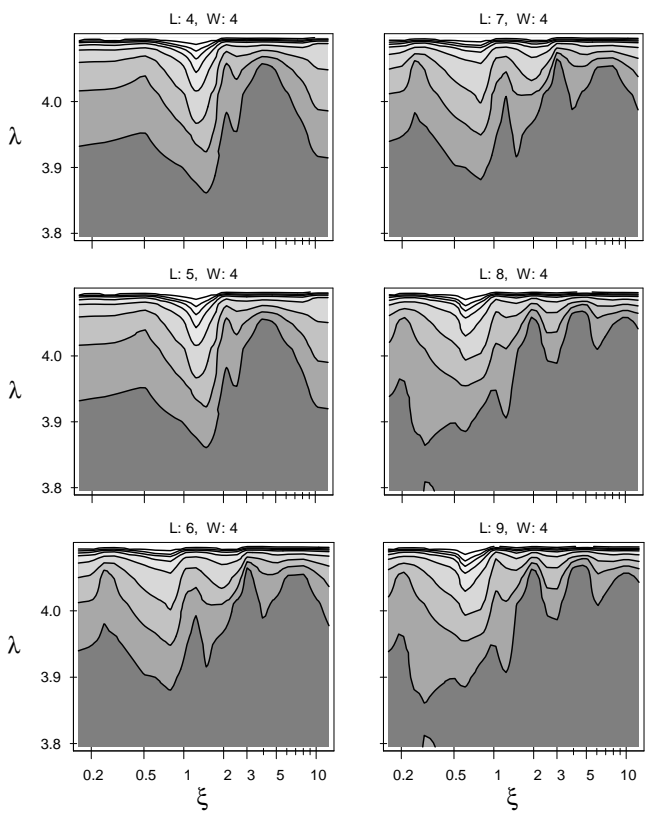

FIG. 7: As Fig. 6, but for $W=4$.

transmitted plane wave field given by the coefficient vector $\boldsymbol{t}=\boldsymbol{T}_{\mathrm{gf}} \boldsymbol{c}_{-}$is

$$
V(x, y)=\frac{1}{\sqrt{D}} \sum_{p=-\infty}^{\infty} \chi_{p}^{-1 / 2} t_{p} e^{i\left(\alpha_{p} x+\chi_{p} y\right)},
$$

which has a total upward energy flux of

$$
\varepsilon_{\mathrm{gf}}=\sum_{p \in \Omega_{f}}\left|t_{p}\right|^{2}=\sum_{p \in \Omega_{f}}\left|T_{p 1}^{\mathrm{gf}}\right|^{2}
$$

since $t_{p}=T_{p 1}^{\mathrm{gf}}$.

We seek to approximate this field by a Gaussian beam of width $v$ given by

$$
V^{\prime}(x, y)=\frac{1}{\sqrt{D}} \sum_{p \in \Omega_{f}} A \chi_{p}^{-1 / 2} B_{p}\left(y_{0}, v\right) e^{i\left[\alpha_{p} x+\chi_{p} y\right]}
$$

where

$$
B_{p}\left(y_{0}, v\right)=\frac{\sqrt{\pi} v}{D} \chi_{p}^{-1 / 2} e^{-v^{2}\left(\alpha_{p}-\alpha_{0}\right)^{2} / 4} e^{-i \chi_{p} y_{0}},
$$

which follows from Fourier transformation. Here the beam waist has been placed on the symmetry line of the structure $x=0$. We include only propagating order terms since the series representing the Gaussian beam converges rapidly.

There are three parameters to be fitted, $y_{0}$, the location of the waist of the Gaussian beam, $v$, its width, and $A$ which is related to the beam amplitude. We first establish the 
magnitude of $A$ by ensuring that both the transmitted field $\boldsymbol{t}$ and its Gaussian approximation carry the same flux. This constrains $|A|$ according to

$$
\sum_{p \in \Omega_{f}}\left|t_{p}\right|^{2}=|A|^{2} \sum_{p \in \Omega_{f}}\left|B_{p}\left(y_{0}, v\right)\right|^{2}
$$

Then writing $A=|A| e^{i \theta_{0}}$, where $\theta_{0}$ is the absolute phase of the beam, we set up a least squares problem that corresponds to minimizing the flux of the difference between the transmitted amplitudes and their Gaussian approximates. We thus seek to minimize

$$
F\left(\theta_{0}, y_{0}, v\right)=\sum_{p \in \Omega_{f}}|| A\left|e^{i \theta_{0}} B_{p}\left(y_{0}, v\right)-t_{p}\right|^{2}
$$

using standard nonlinear optimisation techniques, such as are implemented in the Mathematica [25] function FindMinimum. We denote the minimum value by $F_{\min }$, and note that it is constrained to lie in the interval $[0,4]$, given the normalization of the field amplitudes. The lower limit $F_{\min }=0$ corresponds to a perfect fit between the output beam and a Gaussian in both amplitude and phase.

In Fig. 8 we present a typical result from a taper that provides highly efficient coupler between the PCW and free space for PC1. Figures 8(b) and (c) show that the amplitudes of the plane wave coefficients decay rapidly and smoothly. While the data $\left\{A_{p}\right\}$ are discrete and meaningful only for integer $p$, the line has been drawn to distinguish the fit from the data, to aid in their interpretation. Both their magnitudes and their phase can be modelled accurately by a Gaussian beam, as evidenced by the fact that $F_{\min }=0.002$. The waist of the fitted beam lies well inside the taper, 2.6 periods below its mouth.

It is interesting to compare the output of the taper in Fig. 8 with that from a PC waveguide radiating directly into free space, with no tapering at all (Fig. 9). The transmittance now falls from 0.995 to 0.77 . Note in Fig. 9(b) how the intensities of the plane wave coefficients no longer decrease smoothly with order, but rather tend to increase. This can be understood physically since the wave field has to have strong evanescent components to follow the rapidly changing boundary conditions applying near the right-angle corners at the guide-free space boundary. The phase of the coefficients is well-fitted, but the gross non-Gaussian variation of the intensities renders this meaningless, even though $F_{\min }=0.022$ is surprisingly small. Note that the optimum fit has a width $v=0$, and thus in reality resembles a wave radiated by a two-dimensional point source rather than a Gaussian beam. 
(a)

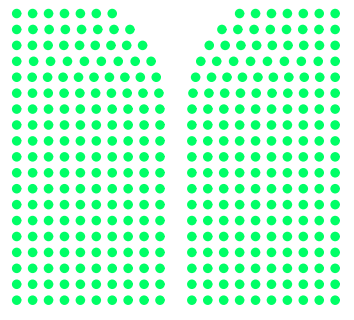

(b)

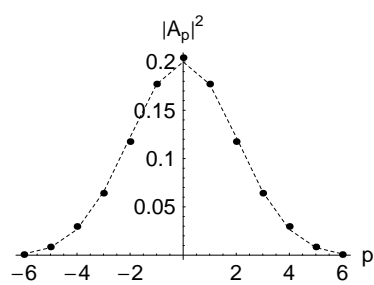

(d)

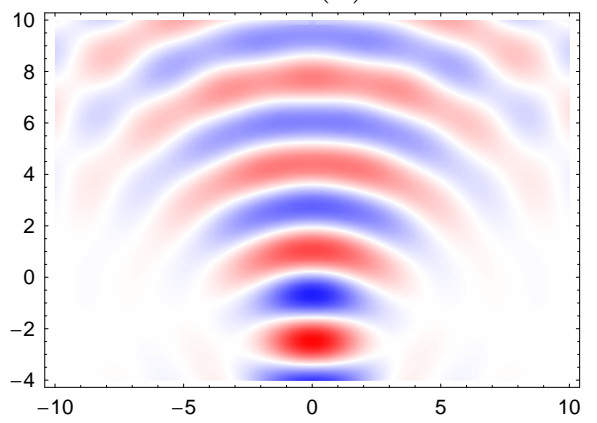

(c)

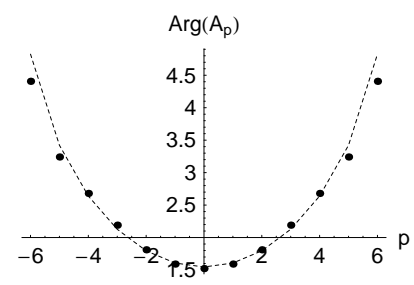

(e)

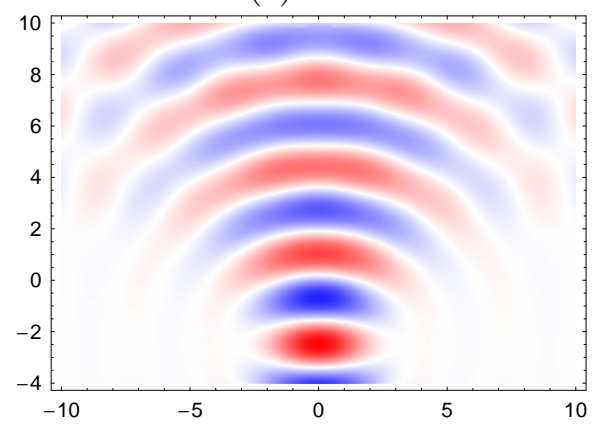

FIG. 8: Analysis of the form of the wave radiated from PC1 with a taper with $W=8, L=7$ layers and $\xi=3$ shown in (a) for $\lambda=3.3 d$. (b) and (c) show the intensity and phase of the plane wave coefficients $A_{p}=t_{p}$ and $A_{p}=A B_{p}$, for the output beam (dots) and its Gaussian fit (dashed line). (d) and (e) respectively show the real part of the output field and its Gaussian fit with $y_{0}=-2.583, v=1.571$ and $F_{\min }=0.002$.

The "beam waist" corresponds to the mouth of the guide, and the fields shown below this correspond to the continuation of the plane wave field from above rather than the actual waveguide field.

Turning now to PC2, we show typical results in Fig. 10 for $W=4, L=7$ and $\xi=3$ and $\lambda=4.08 d$, for which $T=0.985$. The fit between the output beam and a Gaussian form is now worse than for Fig. 8 even though the lower Fourier coefficients match well; not only is $F_{\min }=0.136$ much larger, but also the beam structure has clear non-Gaussian characteristics. For example, in the spatial plots (d) and (e), the radiated beam has a waist, but seems to be asymmetric when the fields above and below the waist are compared. The radiated beam has a core which seems above the waist to have a double structure, and several other weak diffracted beams are evident. All this fine structure cannot be replicated in the Gaussian fit.

For the same data but in the absence of a taper between the guide and free space (Figs 11), 
(a)

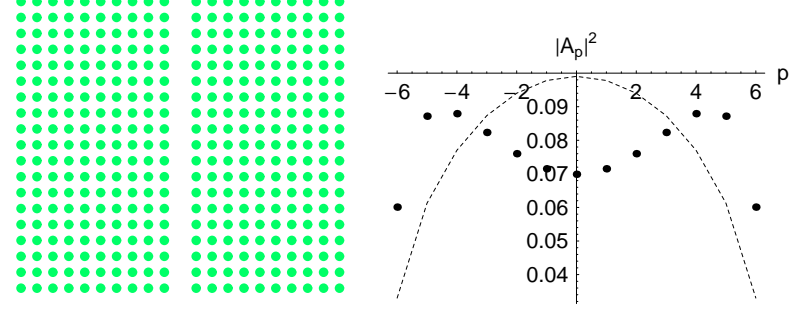

(d)

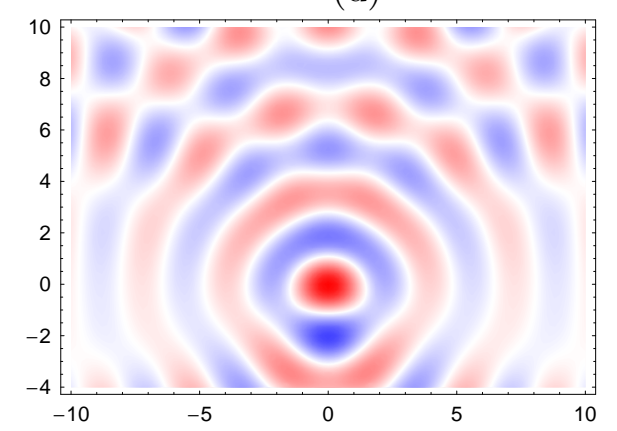

(c)

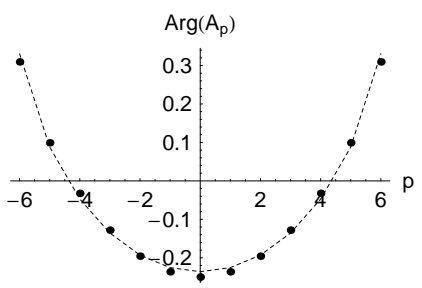

(e)

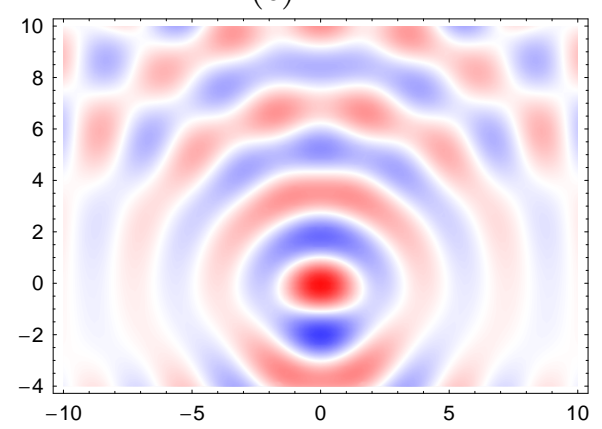

FIG. 9: As Fig. 8, but in the absence of a taper, as in (a). The fitting parameters are $y_{0}=-0.446$, $v=0.000$ and $F_{\min }=0.022$.

(a)

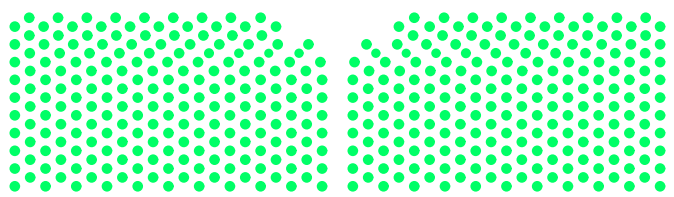

(b)

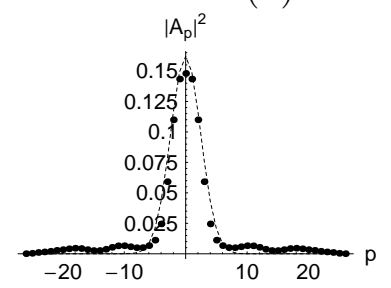

(d)

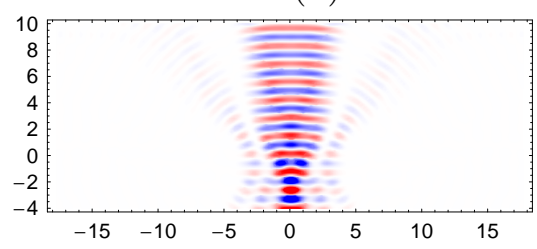

(c)

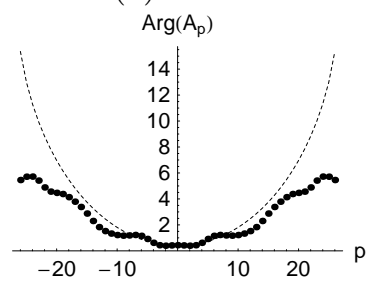

(e)

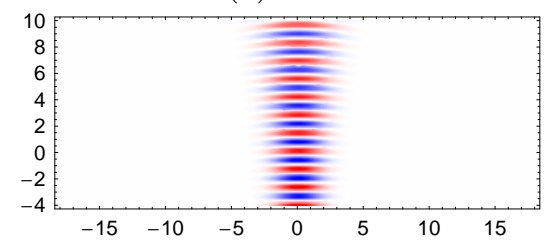

FIG. 10: As Fig. 8 but for PC2 and the other parameters given in the text. The fitting parameters are $y_{0}=-4.233, v=2.341$ and $F_{\min }=0.136$. 
(a)

(b)

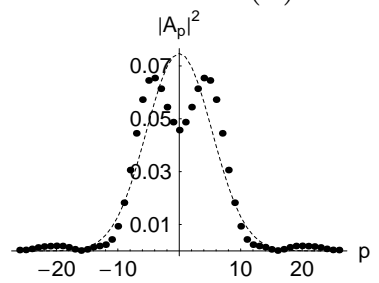

(d)

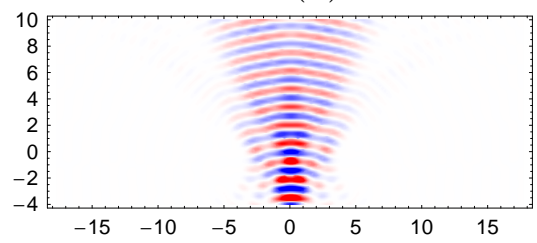

(c)

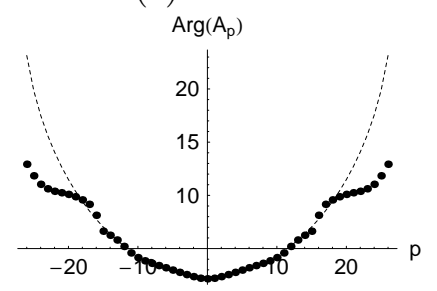

(e)

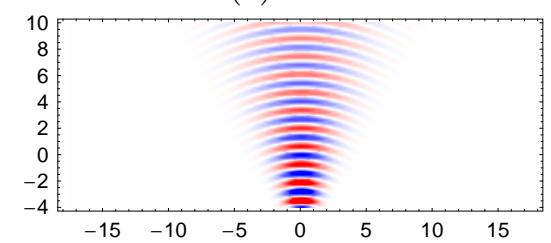

FIG. 11: As Fig. 10 but in the absence of the taper. The fitting parameters are $y_{0}=-5.876$, $v=1.061$ and $F_{\min }=0.061$.

the transmittance is quite good $(0.92 \%)$, but once again the output beam cannot be well represented by a Gaussian beam $\left(F_{\min }=0.061\right)$. The radiated beam has a double structure, which is clear in both the intensity of its plane wave coefficients and its spatial distribution. Once again, the beam radiated is not symmetric about its waist, and is much more divergent than in the presence of the taper. It is interesting to compare Fig. 11 with Fig. 9; in the case of the hexagonal array, the "flaring" of the waveguide caused by the different positions of cylinders in the last two layers largely weakens the rapid field variations at the waveguide mouth. This is evident in the drop-off of $\left|A_{p}\right|^{2}$ with $|p|$, and as well in the smaller angular divergence of the radiated beam.

\section{COUPLING A GAUSSIAN BEAM INTO A PCW}

Photonic crystal circuits in general consist of waveguides with incorporated optical processing elements, with a Gaussian beam feeding the circuit, and a Gaussian beam desirably being radiated at the circuit output. It is desirable that both input and output junctions be designed to produce low-reflection coupling, and advantageous if the junctions be able to 
function bi-directionally. This leads to the concept of reciprocal couplers, equally adapted to coupling a Gaussian beam from a uniform material into a PCW with low reflection losses, and from the single mode in the PCW back into the uniform material. The Reciprocity Theorem [17] indicates that this is possible if, for example, the PCW at a given wavelength radiates a beam which is very close to Gaussian, as we now show.

We consider the time reversed problem and return the output plane wave field $\boldsymbol{t}$ from the previous section as an incident field into the wave guide. Time reversal requires that the beam amplitudes are conjugated, and we further scale these to ensure that there is unit incident flux. We thus define an incident beam $\boldsymbol{\delta}^{\prime}$, where, using an overbar for complex conjugation $\delta_{p}^{\prime}=\bar{t}_{p} / \sqrt{\varepsilon_{\mathrm{gf}}}$. Using reciprocity we then find that

$$
c_{1}^{(-)}=\sum_{p}\left|T_{1 p}^{\mathrm{fg}}\right|^{2} / \sqrt{\varepsilon_{\mathrm{gf}}}=\sqrt{\varepsilon_{\mathrm{gf}}}
$$

from the definition of $\varepsilon_{\mathrm{gf}}(20)$. Thus the transmitted flux $\varepsilon_{\mathrm{fg}}=\left|c_{1}^{(-)}\right|^{2}=\varepsilon_{\mathrm{gf}}$ is identical to the flux transmitted from the waveguide into free space $\varepsilon_{\mathrm{gf}}-\mathrm{a}$ result which depends on the waveguide having only a single propagating mode.

Of course, in practice, one requires a coupler that can function bi-directionally over a significant wavelength range. It is desirable that the coupling into the waveguide from free space not require an adjustment of beam parameters for each new wavelength. Thus, we investigate whether a given taper structure can couple efficiently a Gaussian with fixed width and waist position over a significant range of wavelengths. Note that in practice the optical system illuminating the PCWG might generate Gaussian beams whose widths and waist positions may be functions of wavelength. However, in order that we may study a situation here independent of specific system characteristics, we consider the illuminating Gaussian beam to have wavelength-independent characteristics.

We start with $E_{z}$ polarization, and the efficient rod-type coupler of Fig. 8. For this structure, we show in Table I the parameters of the optimized Gaussian fits to the beam radiated by the PCW into free space for the wavelengths $3.01 d<\lambda / d<3.40 d$. We take the output beam parameters for the five central wavelengths in Table I and make a global Gaussian best-fit to the whole set of output beams, adjusting the phase of the plane wave coefficient $t_{0}$ to be zero for each wavelength. This best-fit beam has the parameters $y_{0}=$ $-2.869, w_{0}=1.501$, and the parameter $F_{\min }=0.0134$, giving a close approximation to the radiated beams for all wavelengths $3.01 d<\lambda / d<3.30 d$. In Table I, the last column 


\begin{tabular}{lllll}
$\lambda$ & $y_{0}$ & $w_{0}$ & $\varepsilon_{\text {out }}$ & $\varepsilon_{\text {in }}$ \\
\hline 3.01 & -2.661 & 0.5472 & 0.9850 & 0.8329 \\
3.10 & -3.333 & 1.246 & 0.9968 & 0.9732 \\
3.15 & -3.168 & 1.423 & 0.9979 & 0.9908 \\
3.20 & -2.951 & 1.509 & 0.9978 & 0.9948 \\
3.25 & -2.751 & 1.551 & 0.9970 & 0.9939 \\
3.30 & -2.582 & 1.570 & 0.9951 & 0.9902 \\
3.40 & -2.332 & 1.579 & 0.9770 & 0.9676 \\
\hline
\end{tabular}

TABLE I: Gaussian beam parameters $y_{0}$ and $w_{0}$, and the transmittance $\varepsilon_{\text {out }}$ from the PCWG to free space for different wavelengths for the rod-type $\mathrm{PC}$ taper with $W=8, L=7$ layers and $\xi=3$. The values for the global Gaussian best-fit to the output beams for the wavelengths shown are $\left(y_{0}, w_{0}\right)=(-2.869,1.501)$ and $F_{\min }=0.013$. Parameter $\varepsilon_{\text {in }}$ indicates the efficiency when this Gaussian beam is coupled from free space into the PCWG.

gives the transmittance values $\varepsilon_{\text {in }}$ for this Gaussian beam for coupling from free space into the PCW. These are slightly lower than the transmittance values in the reverse direction in the fitted range, and significantly lower at $\lambda=3.01 d$. Nevertheless, they show that the taper can function bi-directionally with transmittance over $97 \%$, for a wavelength range of $\Delta \lambda / \lambda \simeq 1 / 16 \simeq 7 \%$.

In Table II, we present corresponding results for a air-hole type PC taper with parameters given in the caption. The global Gaussian fit to the output beam is worse for this polarization $\left(F_{\min }=0.111\right)$ than for the rod-type lattice for which $F_{\min }=0.013$. As a result, the transmittance into the waveguide is always at least $3 \%$ below that for the transmittance out from the waveguide, with the difference increasing markedly for long wavelengths, where the Gaussian beam parameters $y_{0}, w_{0}$ are depend strongly on wavelength. The taper here functions bi-directionally with transmittance over $95 \%$ for $\Delta \lambda / \lambda \simeq 1 / 38 \simeq 3 \%$.

\section{CONCLUSION}

We have shown that horn-shaped tapers can provide very good coupling between waveguides in free space and uniform media. The designs work best for rod-type PCs, where the 


\begin{tabular}{lllll}
$\lambda$ & $y_{0}$ & $w_{0}$ & $\varepsilon_{\text {out }}$ & $\varepsilon_{\text {in }}$ \\
\hline 3.8 & -3.033 & 0.7576 & 0.9994 & 0.9605 \\
3.85 & -3.062 & 0.8201 & 0.9995 & 0.9615 \\
3.90 & -3.066 & 0.8924 & 0.9995 & 0.9544 \\
3.95 & -3.064 & 0.9873 & 0.9994 & 0.9376 \\
4.00 & -3.083 & 1.149 & 0.9990 & 0.9043 \\
4.05 & -3.413 & 1.660 & 0.9974 & 0.8391 \\
4.08 & -4.233 & 2.340 & 0.9850 & 0.7642 \\
\hline
\end{tabular}

TABLE II: As for Table I, but now with a taper with $W=4, L=7$ layers and $\xi=3$ in an air-hole PC. The optimal values for the global Gaussian best-fit are: $\left(y_{0}, w_{0}\right)=(-2.631,0.905)$ and $F_{\min }$ $=0.111$.

optimal shape of the taper is not a strong function of wavelength or PC parameters. Very advantageously, tapers in rod-type PCs which provide good coupling also radiate beams which are essentially Gaussian. We have verified this not only for the curved tapers described in Sections III-V, but also for efficient linear couplers $(\xi=1)$. This has two advantages for systems whose design requires Gaussian input and output beams: first, the same design can be used on the input and output ends of the waveguide, and second, it is a much simpler design problem to optimize the radiation from the waveguide into free space than in the other direction (there being two fewer parameters to choose - the beam waist and its position). In circumstances where the waveguide radiates a near-Gaussian beam whose parameters do not vary sensitively with wavelength, waveguide-free space designs will provide the fastest route to couplers for Gaussian beams into a PC waveguide.

For hole-type PCs, we have shown that the optimization of coupling from the PC waveguide into free space is much more delicate, with high transmittances over a broad bandwidth being harder to obtain than for rod-type PCs, and output beams being less close approximations to Gaussians. Thus, it is much harder to obtain good bi-directional coupling from compact hole-type tapers.

The treatment given here, based on two-dimensional simulations, is likely to apply to fully three-dimensional geometries as well. Assuming that the waveguide itself has been designed to minimize or eliminate radiation losses, the tapers, which typically would have 
a length of only a few micrometers, are unlikely to increase these significantly. First, by construction the taper is adiabatic, having no abrupt features. Second, changing the width of the waveguide changes the mode's local propagation constant but this is unlikely to change the rate of out-of-plane radiation dramatically.

Perhaps the most important conclusion is that in designing PC waveguide circuits, the guiding principle is to ensure a smooth waveguide wall throughout transitions (see also Kwan et al [23]). The waveguide fields are guided by the first two rows of cylinders in the waveguide walls, and cylinders further into the PC can be moved around somewhat without significantly detracting from coupling efficiencies. This conclusion has been verified in the present two-dimensional study, and should also be tested in the context of particular three-dimensional designs.

This work was produced with the assistance of the Australian Research Council under the ARC Centres of Excellence Program.

\section{Appendix: Profile of Hexagonal Lattice Tapers}

The construction of smooth tapers in hexagonal lattices is difficult, and here we describe the method we have adopted. We distinguish two cases:

1. Each cylinder row is completely inside a grating layer, which occurs when the normalized radius $a$ satisfies $a / d<0.25$.

2. When $a / d>0.25$ the cylinder rows interpenetrate as in Figs. 12 and 13 .

\section{Case 1: cylinder rows do not interpenetrate}

Without interpenetration between the cylinder rows, inside each grating layer, the positions of the cylinder inclusions can be set without constraint with regard to the adjacent layers, and the values $w(y)$ from (18) can be used. For layers corresponding to even numbers $n(0 \leq n \leq L)$, the distance between the centres of the inclusions on the taper wall is exactly $w\left(n d_{y}\right) d_{x}$. However to replicate the variation of the width of the untapered waveguide for layers with odd $n$, the distance between the centres of the inclusions on the taper wall is $\left(w\left(n d_{y}\right)+1\right) d_{x}$. Once the positions of the inclusions on the taper opening are determined, the remaining PC inclusions can be distributed using the technique from Section 2.2. 


\section{Case 2: the cylinder rows interpenetrate}

The inclusions in a grating layer now cannot be distributed independently of adjacent layers because inclusions may overlap. We give precedence to the even numbered layers because their inclusions are closer to the waveguide and we place these inclusions as for Case 1 above. For the odd numbered layers, where each inclusion is to be placed in the middle of four surrounding cylinders from the adjacent layers (or two in the last layer), as in Figs 12 and 13), we use the rule $x_{0}=\left(x_{1}+x_{2}+x_{3}+x_{4}\right) / 4$ where $x_{0}$ is the $x$-coordinate of the inclusion and $x_{i}, i=1,2,3,4$, are the $x$-coordinates of the surrounding cylinders. If the inclusions still overlap, the radius of the inclusion in the odd numbered layer is reduced (see Fig. 13), or this inclusion may be removed.
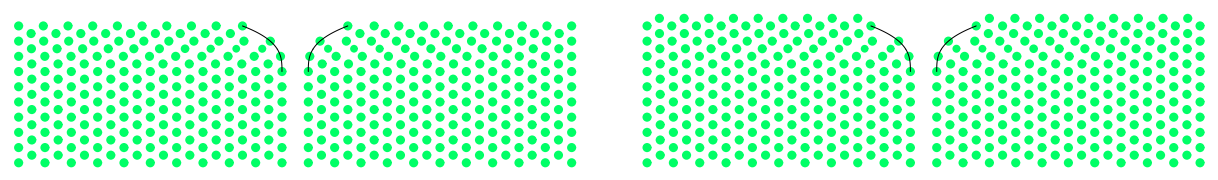

FIG. 12: Left: Taper of length $L=6$ layers, $W=4$ and $a / d=0.3$ in PC2. Right: similar, but for $L=7$ layers.

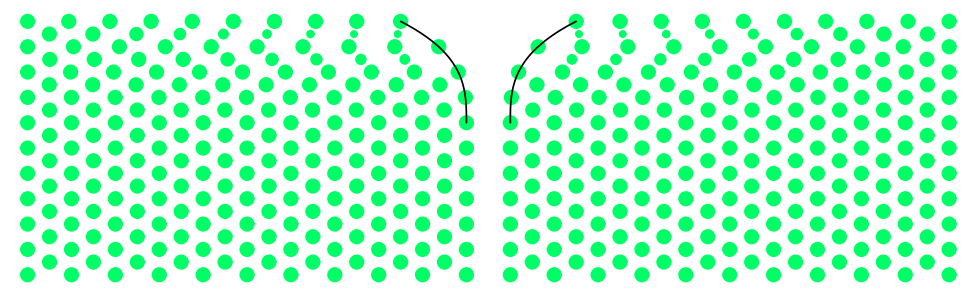

FIG. 13: Taper of length $L=8$ layers, $W=4$ and $a=0.3 d$ in PC2. The radii of some odd layer cylinder have been reduced to $a=0.16 d$ to avoid overlap with other cylinders. 
[1] L. C. Botten, T. P. White, A. A. Asatryan, et al., Opt. Lett. 28, 854 (2003).

[2] A.P. Mekis and J.D Joannopoulos, J. Lightwave Technol, 19, 861 (2001).

[3] Y. Xu, R. K. Lee, and A. Yariv, Opt. Lett. 25, 755 (2000).

[4] T. D. Happ, M. Kamp, and A. Forchel, Opt. Lett. 26, 1102 (2001).

[5] E. Moreno, D. Erni, and C. Hafner, Phys. Rev. E 66, 036618 (2002).

[6] Ph. Lalanne and A. Talneau, Opt. Expr. 10, 354 (2002).

[7] P. Sanchis, J. Marti, A. Martinez, et al., Electron. Lett. 38, 961 (2002).

[8] P. Sanchis, J. Marti, A. Martinez, et al., Opt. Expr. 10, 1391 (2002).

[9] A. R. Weily, K. P. Esselle, and B. C. Sanders, Phys Rev E 68, 016609 (2003).

[10] P. Pottier, I. Ntakis, and R. M. de la Rue, Opt. Comm. 223, 339 (2003).

[11] P. Bienstman, S. Assefa, S. G. Johnson, et al., J. Opt. Soc. Am. B 20, 1817 (2003).

[12] A. Talneau, M. Agio, C. M. Soukoulis, et al., Opt. Lett. 29, 1745 (2004).

[13] P. Sanchis, P. Bienstman, B. Luyssaert, et al., J. Quantum Electron. 40, 541 (2004).

[14] M. Ayre, T.J. Karle, L.Wu, et al., IEEE J. Sel Areas in Comm. 23, 1390 (2005).

[15] P. Kramper, M. Agio, C.M. Soukoulis, et al., Phys. Rev. Lett. 92, 113903 (2004).

[16] S. G. Johnson, P. Bienstman, M.A. Skorobogatiy, et al., Phys. Rev. E 66, 066608 (2002).

[17] R. Petit (ed.), Electromagnetic Theory of Gratings, Chapter 1 (Springer-Verlag, Berlin, 1980).

[18] L. C. Botten, T. P.White, C. M. de Sterke, et al., Optics Express 8, 1592 (2004).

[19] L. C. Botten, T. P. White, A. A. Asatryan, et al, Phys. Rev. E 70, 056606 (2004).

[20] L. C. Botten, N. A. Nicorovici, A. A. Asatryan, et al. R. C. McPhedran, C. M. de Sterke, and P. A. Robinson, J. Opt. Soc. Am. A 17, 2165 (2000).

[21] L.C. Botten, R.C. McPhedran, C.M. de Sterke, et al., Chapter 2 in Electromagnetic Theory and Applications for Photonic Crystals, ed. K. Yasamoto (CRC Press, 2005).

[22] K.Dossou, M.A. Byrne and L.C. Botten, Finite Element Computation of Grating Scattering Matrices and Application to Photonic Crystal Band Calculations, in preparation.

[23] K.-C Kwan, X. Zhang, Z.-Q. Zhang, et al., Appl. Phys. Lett. 82, 4414 (2003).

[24] J. D. Joannopoulos, R .D. Meade and J. N. Winn, Photonic Crystals: Molding the Flow of Light (Princeton U.P., 1995).

[25] S. Wolfram, The Mathematica Book (Wolfram Media, Champaign, Ill.,2003). 